\title{
ON ELATIONS IN SEMI-TRANSITIVE PLANES
}

\author{
N.L. JOHNSON \\ Department of Mathematics \\ The University of Iowa \\ Iowa City, Iowa 52242 \\ U.S.A.
}

(Received January 2, 1980)

ABSTRACT. Let $\pi$ be a semi-transitive translation plane of even order with reference to the subplane $\pi_{0}$. If $\pi$ admits an affine elation fixing $\pi_{0}$ for each axis in $\pi_{0}$ and the order of $\pi_{0}$ is not 2 or 8 , then $\pi$ is a Hall plane. KEY WORDS AND PHRASES. Elations, Semi-transitive planes. 1980 MATHEMATICS SUBJECT CLASSIFICATION CODES. 50005, $05 B 25$.

\section{INTRODUCTION.}

Kirkpatrick [9] and Rahilly [10] have characterized the Hall planes as those generalized Hall planes of order $q^{2}$ that admit $q+1$ central involutions.

In [7] the author has shown that the derived semifield planes of characteristic $\neq 3$ and order $q^{2}$ are Hall planes precisely when they admit $q+1$ central involutions. This extends Kirkpatrick and Rahilly's work as generalized Hall planes are certain derived semifield planes.

If a translation plane $\pi$ of order $q^{2}$ admits $q+1$ affine elations with distinct axes then the generated group \& contains $\mathrm{SL}(2, \mathrm{q}), \mathrm{S}_{\mathrm{Z}}(\mathrm{q})$ or contains a normal subgroup $\mathrm{N}$ of odd order and index 2 (Hering [5]). In the latter case, little is known about \& except that it is usually dihedral. 
In this article, we study semi-transitive translation planes of order $q^{2}$ that admit $q+1$ affine elations.

In [8], the author introduces the concept of the generalized Hall planes of type 1. These are derivable translation planes that admit a particular collineation group which is transitive on the components outside the derivable net. In this situation the group is generated by Baer collineations.

More generally, Jha [6] has considered the "semi-transitive" translation planes.

(1.1) Let $\pi$ be a translation plane with subplane $\pi_{0}$. If there is a collineation group \& such that

1) \& fixes $\pi_{0} \cap \ell_{\infty}$ pointwise,

2) leaves $\pi_{0}$ invariant, and

3) acts transitively on $\ell_{\infty}-\pi_{0} \cap \ell_{\infty}$, then $\pi$ is said to be a semi-transitive translation plane with reference to $\pi_{0}$ and with respect to $\&$.

Our main result is that semi-transitive planes of order not 16 or 64 that admit elations with axis $\mathcal{L}$ fixing $\pi_{0}$ for every component $\mathcal{L}$ of $\pi_{0}$ are Hall planes. We also give a necessary and sufficient condition that a translation plane of order $q^{2} \neq 64$ admitting $q+1$ elations with distinct axes is derivable. 2. TRANSLATION PLANES OF EVEN ORDER $q^{2}$ ADMITTING $q+1$ ELATIONS.

(2.1) THEOREM. Let $\pi$ be a translation plane of even order $q^{2} \neq 64$ that admits $q+1$ affine elations with distinct axes. Let $\eta$ denote the net of degree $\mathrm{q}+1$ that is defined by the elation axes and assume the group $D$ generated by these elations leaves $\eta$ invariant. Then $\eta$ is derivable if and only if $D$ is either isomorphic to $\operatorname{SL}(2, q)$ or is dihedral of order $2(q+1)$ where the cyclic stem fixes at least two components.

PROOF. If $D$ is isomorphic to $\operatorname{SL}(2, q)$ then $n$ is derivable and actually $\pi$ is Desarguesian by Foulser-Johnson-0strom [3].

Let $D=\left\langle\sigma, x \mid \sigma^{2}=\chi^{q+1}=1, \sigma x=x^{-1} \sigma\right\rangle$. If $\langle x\rangle$ fixes the components $x=0$, $Y=\theta$ then we may choose coordinates so that $\sigma$ is $(x, y) \rightarrow(y, x)$ and $X$ is $(\mathrm{x}, \mathrm{y}) \rightarrow\left(\mathrm{xT}_{\mathrm{y}} \mathrm{yT}^{-1}\right)$ for some matrix $\mathrm{T}$ of order $\mathrm{q}+1$. 
By Ostrom [11], Theorem 3, there is a Desarguesian plane $\sum$ containing the two $x$-fixed components and $\eta$. Clearly $\eta$ is an Andre net in $\Sigma$ and thus derivable in $\pi$.

Conversely, suppose $\eta$ is derivable. Since each elation fixes $\eta$, $D$ must fix each Baer subplane of $\gamma$ incident with $\vartheta$. By Foulser [2], Theorem 3, $D \leq G L(2, q)$ in its action on $\pi$ so that $D \leq S L(2, q)$ (each elation is then in $\mathrm{SL}(2, \mathrm{q}))$. By Gleason [4], $\mathrm{D}$ is transitive on the elation axes so $\mathrm{q}+1|| \mathrm{D} \mid$. Thus, $\mathrm{D}$ is clearly $\dot{S L}(2, q)$ or is dihedral of order $2(q+1)$. Moreover, if $\eta$ is derivable then $x$ fixes at least two infinite points of $\pi-\eta$. Let $\bar{n}$ replace $\eta$ so \& fixes $\bar{n}$ componentwise in the derived plane $\bar{\pi}$. Let $\langle\bar{x}\rangle \triangleleft\langle x\rangle$ such that $|\bar{x}|$ is a prime 2-primitive divisor of $q^{2}-1$ (one exists since $q^{2} \neq 64$ ). Then $\bar{\chi}$ fixes at least two infinite points of $\bar{\pi}-\bar{\eta}$ so there is a unique Desarguesian plane $\Sigma$ containing the $\bar{x}$-fixed components of $\bar{\pi}$ (see Ostrom [11], Cor. to Theorem 1-uniqueness comes from the fact that the degree of $\sum \cap \bar{\pi}$ is greater than $q+1)$. Since \& permutes the components of $\sum \cap \bar{\pi}$ (i.e., $\langle\bar{x}\rangle$ is characteristic in $\langle x\rangle), \&$ is a collineation group of $\Sigma$. The collineation $x$ has the form $(x, y) \rightarrow\left(x^{\phi} a, y^{\phi} a\right)$ where $\phi$ is an automorphism of $G F\left(q^{2}\right)$ and $a \in G F\left(q^{2}\right)$. (Note $x$ fixes $\bar{\eta}$ componentwise.) since $q+1$ is odd, $\left\langle\chi^{2}\right\rangle=\langle x\rangle$. Choosing coordinates so that the components of $\bar{\eta}$ are $\mathrm{x}=\theta, \mathrm{Y}=\theta, \mathrm{y}=\mathrm{x} \alpha, \alpha \in \mathrm{GF}\left(\mathrm{q}^{2}\right)$ then $x$ fixes $y=x \alpha$ for all $\alpha \in G F\left(q^{2}\right)$ if and only if $\alpha^{\phi}=\alpha$. Since $\left\langle\chi^{2}\right\rangle=\langle x\rangle$, we may assume $\phi=1$. Thus, $\chi$ fixes $\ell_{\infty}$ of $\Sigma$ pointwise. Since $\Sigma$ and $\pi$ share at least two components (those fixed by $\bar{x}$ ), $\chi$ must fix at least two components of $\pi$.

\section{SEMI-TRANSITIVE TRANSLATION PLANES OF EVEN ORDER.}

Let $\pi$ be a translation plane of even order $q^{2}$ that admits $q+1$ elations as in section 2. Then, $\pi$ is a derivable plane provided the generated group D is dihedral and the cyclic stem fixes at least 2 points or $S L(2, q)$. In any case let $\eta$ denote the net defined by the elation axes. Let \& be a collection group that commutes with D. Then clearly, \& must fix $\eta \cap \ell_{\infty}$ pointwise. (3.1) THEOREM. Let $\pi$ be a translation plane of even order $q^{2} \neq 64$ that admits $q+1$ elations with distinct axes. Assume the group $D$ generated by these 
$q+1$ elations leaves the net $\eta$ of the elation axes invariant. Let \& be a collineation group which commutes with $D$ and is transitive on $\ell_{\infty}-\eta \cap \ell_{\infty}$. Then $\pi$ is a Hall plane.

PROOF. Since $q^{2} \neq 64$, there is a prime 2-primitive divisor $m$ of $q^{2}-1$. By Gleason [4], $q+1|| D \mid$. Clearly, $m \mid q+1$. Let $x$ be an element of $D$ of order $m$. $x$ acts on the $q(q-1)$ points of $\ell_{\infty}-\eta \cap \ell_{\infty}$ so must fix at least two points of $\ell_{\infty}-\eta \cap \ell_{\infty}$. Since \& commutes with $x$ and \& is transitive on $\ell_{\infty}-\eta \cap \ell_{\infty}, \quad x$ must fix $\ell_{\infty}-\eta \cap \ell_{\infty}$ pointwise.

By the corollary to Theorem 1, Ostrom [11], there is a Desarguesian plane $\Sigma$ such that the components fixed by $\chi$ in $\pi$ are exactly the common components of $\Sigma$ and $\pi$. Let $\pi=\eta \cup m$ where $m$ is the net complementary to $\eta$ in $\pi$. Then $\Sigma=\bar{\gamma} \cup m$ for some net $\bar{\eta}$ of degree $q+1$. So $\Sigma$ and $\pi$ are two extensions of a net $m$ of critical deficiency (see 0strom [12]). Then $\pi$ must be Hall since $\Sigma$ and $\pi$ must be related by derivation (i.e., $\pi$ cannot be itself Desarguesian) by Ostrom [12].

The conditions of (3.1) are close to giving the definition of a "semi-transitive" translation plane (see (1.1)). In (3.1), it is possible that \& may not satisfy condition 2. Also, it is not clear that a semi-transitive translation plane is derivable. However, Jha [6] shows if $\pi$ has order not 16 and there is a nontrivial kern homology in $\pi$ then $\pi$ is derivable and $\pi_{0}$ is a Baer subplane.

We may overcome this restriction on the kern in our situation:

(3.2) THEOREM. Let $\pi$ be a semi-transitive translation plane of even order with respect to a collineation group \& and with reference to a subplane $\pi_{0}$. Let $\pi$ admit an affine elation for each axis in $\pi_{0}$.

1) If the order of $\pi_{0}$ is not 8 then $\pi$ is derivable.

2) If the order of $\pi_{0}$ is not 2 or 8 then $\pi$ is a Hall plane.

PROOF. Following Jha's [6] ideas, let $\pi_{1}$ be a minimal subplane of $\pi$ properly containing $\pi_{0}$. Clearly, the stabilizer $\&_{1}$ of $\pi_{1}$ is a semi-transitive collineation group of $\pi_{1}$ with reference to $\pi_{0}$. Moreover, a sylow 2-subgroup of $\xi_{1}$ must leave $\pi_{0}$ pointwise fixed since \& fixes $\pi_{0}$ and fixes $\pi_{0} \cap \ell_{\infty}$ pointwise. (Note $\left|\&_{\pi_{1}}\right|$ is divisible by $\left(2^{r}+1\right)-\left(2^{s}+1\right)$ for some $\left.r, s.\right)$ Clearly, $\pi_{0}$ is a Baer subplane of $\pi_{1}$. 
Every elation which leaves $\pi_{0}$ invariant must also leave any superplane invariant. So the group $D$ generated by the elations leaves $\pi_{1}$ invariant and, clearly, \& commutes with $D$ since \& fixes $\pi_{0} \cap \ell_{\infty}$ pointwise (\& must commute with each central collineation fixing $\pi_{0}$ ).

By (3.1), if the order of $\pi_{0}$ is not 8 then $\pi_{1}$ is a Hall plane and $\pi_{1}$ is derivable. We may now directly use Jha [6] to show that if the order of $\pi_{0}$ is not 2 then $\pi_{1}=\pi$ (that is, Jha uses the hypothesis that there is a kern homology to show that $\pi_{1}$ is derivable).

Actually, our proof of (3.2) proves the following more general theorem for arbitrary order.

(3.3) THEOREM. Let $\pi$ be a semi-transitive translation plane with reference to $\pi_{0}$ and with respect to \& and order $\mathrm{p}^{\mathrm{r}}$. Let $\mathrm{x}$ be a collineation generated by central collineations leaving $\pi_{0}$ invariant such that $|x|$ is a prime p-primitive divisor of (order $\left.\pi_{0}\right)^{2}-1$ (where the order of $\pi_{0}$ is not 2). Then $\pi$ is a Hall plane.

Note that a semi-transitive plane of odd order $\mathrm{p}^{2 \mathrm{r}}$ must admit Baer p-elements (see Jha [6]). By Foulser [1], we could then not have both Baer p-elements and elations so we could restate our Theorem (3.2) without reference to order. (3.2)2) is also valid if the order $\pi_{0}$ is 8 . The arguments supporting this will appear in a related article.

\section{REFERENCES}

1. Foulser, D. A. Baer p-elements in translation planes, J. Algebra 31(1974) 354-366.

2. Foulser, D. A. Subplanes of partial spreads in translation planes, Bu11. London Math. Soc. 4(1972) 1-7.

3. Foulser, D. A., N. L. Johnson and T. G. Ostrom. Characterization of the Desarguesian and Hall planes of order $\mathrm{q}^{2}$ by $\operatorname{SL}(\mathrm{q}, 2)$, submitted.

4. Gleason, A. M. Finite Fano planes, Amer. J. Math. 78(1956) 797-806.

5. Hering, Ch. On shears of translation planes, Abh. Math. Sem. Hamburg 27(1972) 258-268.

6. Jha, V. Finite semi-transitive translation planes, to appear.

7. Johnson, N. L. On central collineations of derived semifield planes, J. Geom. $11 / 2(1978)$ 139-149. 
8. Johnson, N. L. Distortion and generalized Hall planes, Geom. Ded. 4(1975) $1-20$.

9. Kirkpatrick, P. B. A characterization of the Hall planes of odd order, Bull. Austral. Math. Soc. 6(1972) 407-415.

10. Rahilly, A. Finite collineation groups and their collineation groups, Thesis, Univ. of Sydney, 1973.

11. Ostrom, T. G. Linear transformations and collineations of translation planes, J.Algebra (3) 14(1970) 405-416.

12. Ostrom, T. G. Nets with critical deficiency, Pacific J. Math. 14(1964), 1381-1387. 


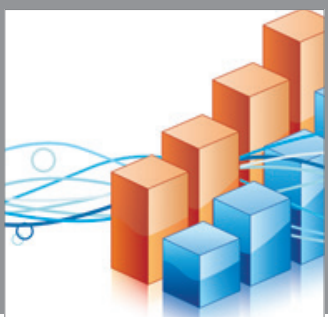

Advances in

Operations Research

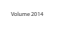

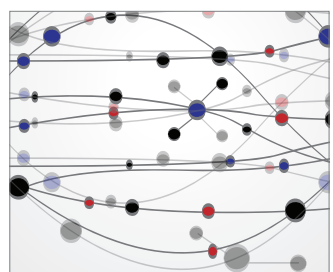

\section{The Scientific} World Journal
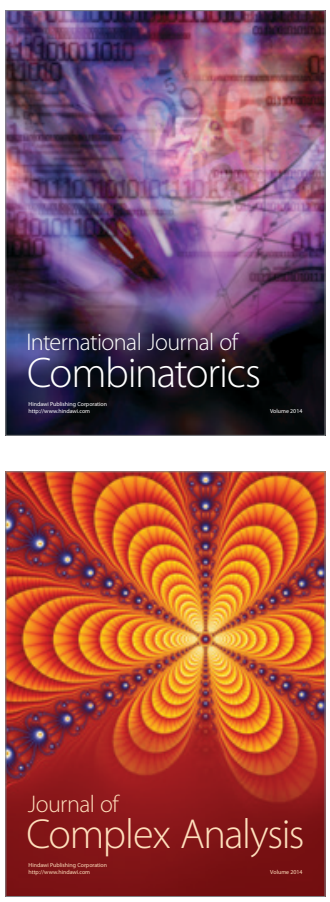

International Journal of

Mathematics and

Mathematical

Sciences
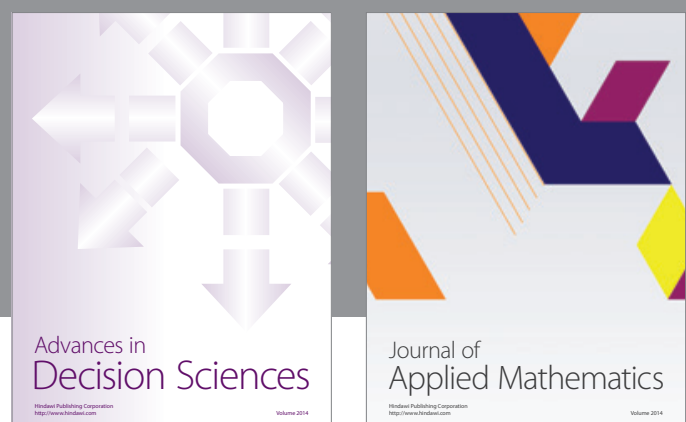

Journal of

Applied Mathematics
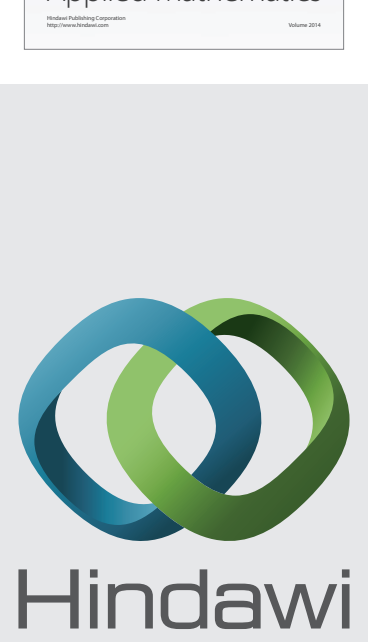

Submit your manuscripts at http://www.hindawi.com
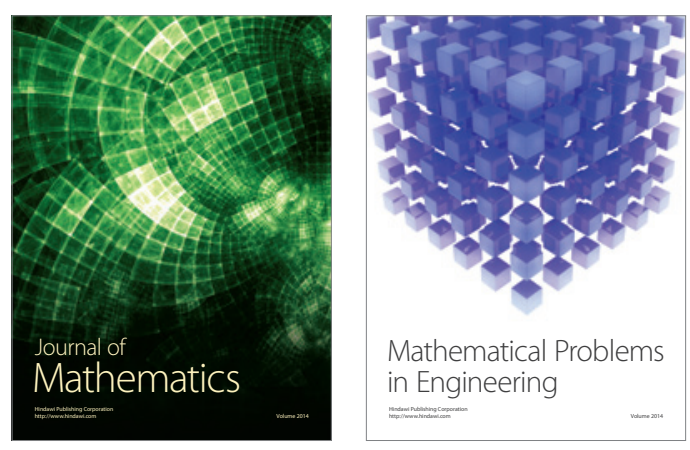

Mathematical Problems in Engineering
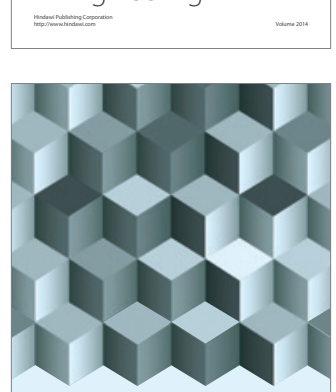

Journal of

Function Spaces
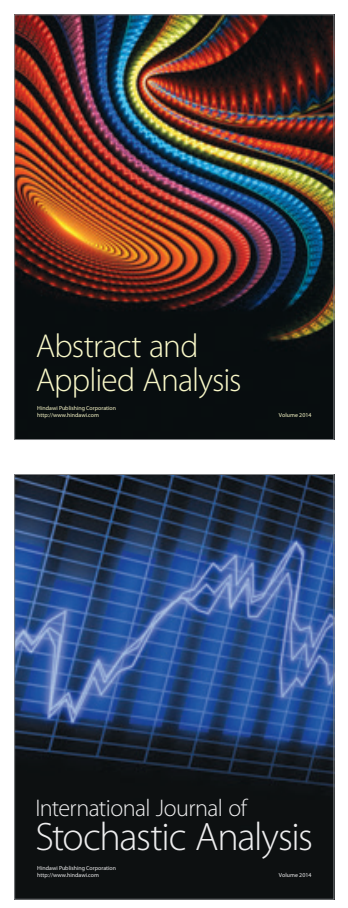

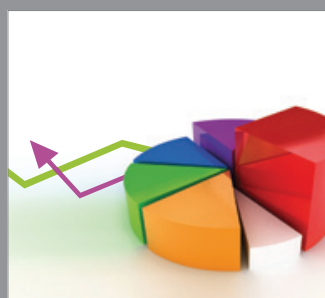

ournal of

Probability and Statistics

Promensencen
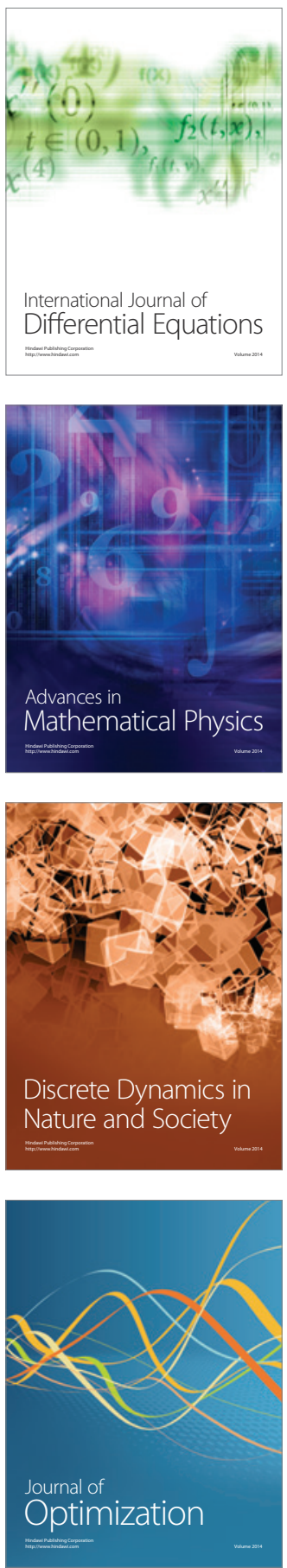